\title{
Doença periodontal e asma: revisão da literatura
}

\author{
Periodontal disease and asthma: literature review
}

\author{
Kaliane Rocha Soledade Marques ${ }^{1}$, Adelmir Souza-Machado ${ }^{2}$ \\ ${ }^{1}$ Mestranda em Processos Interativos dos Órgãos e Sistemas, Instituto de Ciências da Saúde - Universidade \\ Federal da Bahia. ${ }^{2}$ Professor Adjunto do Departamento de Biomorfologia do Instituto de Ciências da Saúde - \\ Universidade Federal da Bahia.
}

Resumo

Objetivo: Revisar a literatura a respeito da possível relação entre a presença de asma e o desenvolvimento da doença periodontal. Metodologia: Foi realizada uma revisão consultando as bases de dados Medline e Pubmed usando os seguintes descritores: "periodontal disease", "oral hygiene", "asthma". Dos artigos científicos identificados, foram selecionados 35 para compor a revisão. Síntese dos dados: A infecção periodontal pode influenciar na hiper-resposta inflamatória em pacientes com asma, através de alterações imunológicas, além de servirem como fontes de microrganismos para o desenvolvimento de infecções pulmonares. Medicações para o controle da asma como os corticosteróides, podem alterar a capacidade de defesa do organismo, diminuir o fluxo salivar com diminuição do $\mathrm{pH}$ da saliva além de contribuir para o aumento do metabolismo ósseo, o que pode favorecer o aparecimento ou agravamento da doença periodontal. Conclusões: Estudos mostram uma possível relação entre a asma e desenvolvimento da doença periodontal, porém são necessárias pesquisas de caráter epidemiológico para confirmação desta associação.

Palavras-chave: Perda da Inserção Periodontal. Higiene Bucal. Asma.

\section{Abstract}

Objective: To review the literature regarding the possible relationship between the presence of asthma and the development of periodontal disease. Methods: We conducted a review referring to the Medline database using Pubmed accessing the following keywords: periodontal disease, oral hygiene, asthma. Of the papers identified, 35 were selected to compose the review. Summary of the findings: The periodontal infection may influence the hyper-inflammatory response in patients with asthma through immunological changes, as well as sources of microorganisms for the development of lung infections. Medications for asthma control such as corticosteroids may alter the ability of defense, reducing the saliva flow decreasing the $\mathrm{pH}$ of saliva and contributing to increased bone turnover, which may favor the onset or worsening of periodontal disease. Conclusions: Studies have shown a possible link between asthma and development of periodontal disease, but research is needed to confirm an epidemic of the association.

Keywords: Periodontal Attachment Loss. Oral Hygiene. Asthma.

\section{INTRODUÇÃO}

Tem sido amplamente documentada as relações entre as infecções orais e doenças sistêmicas. A infecção periodontal possui a capacidade de estimular uma resposta imunológica em seu hospedeiro com efeitos sistêmicos importantes (SCANNAPIECO; DASANAYAKE; CHHUN, 2010). Por se tratar de uma doença infecciosa com características imunoinflamatórias altamente discutidas, a doença periodontal parece contribuir para o estabelecimento de infecções do trato respiratório, seja por aspiração de organismos patogênicos, seja pela sensibilização do epitélio desencadeado por reações imunes (BÁGYI et al., 2009). Concomitantemente, pesquisas trazem a discussão sobre a influência exercida por alguns periodontopatógenos na redução da hiper-resposta imunológica de pacientes com histórico de alergias respiratórias e asma.

Recebido: 30 de setembro de 2011; revisado: 20 de dezembro de 2011. Correspondência / Correspondence:Kaliane Rocha Soledade Marques. Endereço: Rua Vênus, №. 191, Bairro Jardim Acácia. CEP: 44.004-416. Feira de Santana, Bahia, Brasil. Tel.: (75) 3021-0140. E-mail: krsoledade@gmail.com.
A asma é uma doença inflamatória crônica das vias aéreas caracterizada por uma hiper-resposta bronquial, limitação reversível do fluxo aéreo e recorrentes episódios de respiração ofegante e curta, opressão torácica e tosse. É considerada uma síndrome complexa, com diversos fenótipos clínicos e inflamatórios. Vários fatores estão relacionados à sua patogênese, incluindo tanto componentes ambientais como genéticos (MORJARIA; POLOSA, 2010). Além disso, algumas medicações utilizadas no tratamento da asma atuam inibindo o sistema imunológico do indivíduo, favorecendo o aparecimento de infecções, ao mesmo tempo em que podem alterar o metabolismo ósseo, com diminuição de sua densidade mineral.

Considerável aumento na prevalência da asma vem sendo mundialmente

observado ao longo das últimas décadas. 0 International Study of Asthma and Allergies in Childhood (ISAAC) possibilitou o conhecimento sobre a prevalência da asma em várias partes do mundo. O estudo realizado em 56 países, durante a sua primeira fase, mostrou uma 
variação na prevalência da doença em torno de 1,6 a $36,8 \%$. No Brasil, a prevalência identificada foi $20 \%$, nível similar ao observado em países desenvolvidos (ISAAC, 1998).

O objetivo deste trabalho é revisar a literatura a cerca da possível contribuição da asma no estabelecimento e/ou progressão da doença periodontal, seja através das alterações inflamatórias induzidas pela doença, seja pelas alterações no metabolismo ósseo provocada pelo uso de corticosteróides.

\section{Imunologia da Doença Periodontal}

As doenças periodontais são causadas pela ação de microrganismos, e podem ser classificadas genericamente em gengivites e periodontites. A gengivite compreende a inflamação das estruturas periodontais de proteção (tecido gengival) e a periodontite é um processo inflamatório que se estende às estruturas periodontais de suporte (ligamento periodontal e osso alveolar) (ARMITAGE, 1999).

A periodontite é uma doença inflamatória complexa e de natureza multifatorial causada principalmente pelo acúmulo de bactérias na superfície dental, formando um biofilme. Seu estabelecimento é determinado pelo desequilíbrio existente entre a microbiota presente na placa bacteriana e a resposta imunológica do hospedeiro, levando a destruição dos tecidos de suporte do dente. Por se tratar de uma doença com etiologia multibacteriana, seu diagnóstico baseado em dados microbiológicos é extremamente difícil (PACE; McCULLOUGH, 2010).

Bactérias Gram-negativas presentes no biofilme dental liberam uma variedade de produtos tóxicos, biologicamente ativos, como as endotoxinas bacterianas conhecidas como lipopolissacarídeos (LPS), proteínas tóxicas, peptídeos quimiotáticos, e ácidos graxos orgânicos. Estas moléculas iniciam uma resposta inflamatória local, e quando não tratada, pode evoluir para uma inflamação crônica (ARMITAGE, 1999; PACE; McCULLOUGH, 2010). Com a evolução da doença, podese gerar a perda de inserção devido à destruição do tecido conjuntivo e ósseo provocado pelas reações inflamatórias e imunológicas ao biofilme.

Entre os patógenos periodontais de maior freqüência encontram-se Aggregatbacter actinomycetencomitans, Porphyromonas gingivalis, Actinomyces isrelii, Capnocytophaga sp., Eikenella corrodens e $P$. intermédia. Atualmente acredita-se que patógenos periodontais são necessários mais isoladamente insuficientes para que a doença periodontal estabeleça-se. A resposta imunológica exibida pelo hospedeiro pode determinar o grau de destruição e forma de disseminação desses patógenos sistemicamente (SOCRANSKY; HAFFAJEE, 1992).

Um dos modelos etiológicos da doença periodontal sugere que a periodontite é mediada por células da resposta imune do Th2. Como resultado temse a ativação das células $B$ e produção de interleucina1, configurando-se um provável mediador de destruição tecidual. Um segundo modelo sugere que células da resposta imune Th1 e IFN-ã estimulam macrófagos e monócitos a produzirem citocinas pró-inflamatórias tais como interleucina-1á, interleucina-1â, interleucina6, interleucina-8, fator de necrose tumoral á (TNFá), além de prostaglandina B2 envolvidas com a reabsorção óssea e destruição do tecido conjuntivo do periodonto (FRIEDRICH et al., 2006; VAN DYKE, 2007). Outros estudos, no entanto, mostram resultados conflitantes. Foram observadas reduções de IL-2 e IFN-ã no soro de pacientes portadores de periodontite (SIGUSH et al., 1998) e uma diminuição na contagem de mastócitos no tecido de pacientes portadores de periodontite crônica justificando a predominância de células Th2 no estabelecimento da doença (GEMMELL; CARTER; SEYMOUR, 2004). O terceiro modelo propõe que diferentes fases da história natural da doença periodontal sejam mediadas por diferentes subtipos de células T. Células Th1 estão associadas com lesões estabelecidas, uma vez que a diferenciação de células $T$ CD4+ em linfócitos Th1 é dependente da IL-12 e se dá em resposta às bactérias intracelulares, vesiculares ou não, alguns parasitas e vírus que podem infectar macrófagos e ativá-los, ou ativar diretamente as células natural killer. Estas células expressam principalmente uma resposta imune celular pró-inflamatória. Já as células Th2 podem exerce o papel mais importante no avanço e progressão das lesões periodontais devido ao seu aspecto antiinflamatório e de resposta imune humoral (ZANATTA et al., 2009). Os diferentes resultados encontrados pelas pesquisas demonstram a inexistência de critérios metodológicos para avaliação.

\section{Doença Periodontal e Doenças Pulmonares}

Apesar do grande número de patógenos periodontais já ter sido identificado, o estabelecimento da doença é uma junção entre a agressividade apresentada pelos microrganismos do biofilme dental, a capacidade de defesa do hospedeiro e modificadores ambientais como o tabagismo (GOMES-FILHO; PASSOS; CRUZ, 2010). A bolsa periodontal pode constituir-se um ambiente propício, adequando temperatura, umidade e suprimento nutricional, não somente para o desenvolvimento de patógenos bucais específicos como também podem abrigar microrganismos reconhecidos como precursores de doenças respiratórias como a $\mathrm{P}$. aeruginosa e bacilos entéricos (AZARPAZHOVH; LEAKE, 2006; PIZZO et al., 2010). Sendo assim, a contaminação da porção final do trato respiratório pode ser causada pela aspiração da saliva contendo tais patógenos. Além disso, bactérias próprias do trato digestivo podem colonizar a região orofaríngea, e serem aspirados para o interior do pulmão. Falhas no mecanismo de defesa do hospedeiro para eliminação destes patógenos da 
superfície respiratória resultam em sua multiplicação e conseqüentemente infecção e destruição tecidual (SCANNAPIECO, 1999).

Estudos têm demonstrado que a qualidade e quantidade da placa dental, principalmente em indivíduos portadores de periodontite, estão fortemente associadas ao desenvolvimento da pneumonia ou podem estar relacionadas à exacerbação dos sintomas de doenças pulmonares obstrutivas crônicas (DPOC) (AZARPAZH; LEAKE, 2006). Uma vez que em $1 \mathrm{~mm}^{3}$ de placa pode conter mais de $10^{6}$ bactérias com 300 espécies aeróbias e anaeróbias facultativas diferentes, alguns estudos apontam que esta combinação de depósitos bacterianos e constituintes salivares formam um reservatório de patógenos respiratórios como o Staphylococcus aureus e Pseudomonas aeruginosa que são capazes de provocar pneumonia e abscessos pulmonares, sugerindo ainda que a quantidade de bactérias aspiradas são mais importantes que o tipo de microorganismo (GOMES-FILHO; PASSOS; CRUZ, 2010).

Em resposta à infecção bacteriana situada no periodonto, as células do tecido epitelial e conjuntivo são as principais responsáveis pela secreção de citocinas. Porém, bactérias orais podem estimular células mononucleares periféricas a produzir citocinas principalmente a IL-1á e TNFá. As células epiteliais são também conhecidas por sua alta expressão de moléculas de adesão celular em resposta a estimulação por citocinas, uma vez que a variação na expressão destas moléculas de adesão pode alterar a interação entre bactérias patogênicas e a superfície da mucosa (SCANNAPIECO, 1999).

A aderência dos patógenos na mucosa respiratória pode ser explicada pelas alterações sofridas pelo epitélio originadas do elevado nível de bactérias proteolíticas periodontais e produção de enzimas como a manoxidase, fucosidase, hexosaminidase e sialidase. Além disso, existe uma relação direta entre a habilidade da saliva em degradar a fibronectina e o nível de higiene oral (GOMES-FILHO; PASSOS; CRUZ, 2010). A perda da fibronectina presente na superfície da mucosa por ação de enzimas e a presença de enzimas hidrolíticas próprias da doença periodontal associada à patógenos, entre eles $O P$. gingivalis e espiroquetas, podem reduzir a habilidade das mucinas em se aderir a patógenos como Haemophilus influenzae, resultando em diminuição das defesas não-específicas do hospedeiro, influenciando no estabelecimento da infecção principalmente em indivíduos de alto-risco (PIZZO et al., 2010). As bactérias $P$. gingivalis, por exemplo, produzem enzimas que degradam estas moléculas salivares bem como possuem a capacidade de produzir outras enzimas que degradam a película salivar expondo os receptores para os patógenos respiratórios (GEMMELL; CARTER; SEYMOUR, 2004).

R. Ci. med. biol., Salvador, v.10, n.3, p.263-269, set./dez. 2011

\section{Asma e Doença Periodontal}

Apesar de a literatura atual trazer informações consistentes quanto ao desenvolvimento de pneumonias e DPOC em portadores de doença periodontal, poucos estudos foram realizados a fim de esclarecer a relação entre asma e o desenvolvimento e progressão de alterações nos tecidos periodontais. A asma pode ser classificada em extrínseca (atópica e não-atópica) e intrínseca. A asma extrínseca está relacionada à predisposição genética para uma resposta do tipo I, ou resposta de hipersensibilidade imediata, contra uma extensa gama de estímulos externos ambientais. Os pacientes que produzem anticorpos IgE para mais de um antígeno são rotulados de atópicos. A asma extrínseca pode também resultar de uma exposição a uma grande variedade de compostos químicos orgânicos ou inorgânicos, como os diisocianatos, e etilenodiamina, os quais podem se manifestar como asma ocupacional, sem que haja participação mediada pela IgE (asma não-atópica) (HUMBERT et al., 1999).

A asma intrínseca ocorre em pessoas sem evidências de atopia. Os testes cutâneos são negativos para alérgenos específicos, sendo os níveis séricos da IgE total normal ou mesmo baixos. A asma intrínseca tem seu início na idade adulta, e se caracteriza por resposta terapêutica insuficiente aos broncodilatadores, necessitar de uso prolongado de corticóides e apresentar um declínio mais rápido nos parâmetros das provas de função pulmonar. As células T destes pacientes secretam IL-5, porém, em comparação aos portadores de asma alérgica, produzem reduzidas quantidades de IL-4. A asma intrínseca está associada a uma ativação anormal Th2 podendo ser considerada como uma reação de hipersensibilidade tardia (MATOS; MACHADO, 2007).

Estudos que buscam uma associação entre asma e doença periodontal têm reportados resultados variados. A variação dos resultados pode ser explicada pela metodologia empregada nos estudos, bem como a escolha dos critérios de associação. Desta forma, a relação entre a asma e doença periodontal tem sido avaliada tanto pelas características inflamatórias próprias das patologias como também através da análise das alterações metabólicas advindas do uso de medicações para o controle da asma.

A hipótese da higiene afirma que uma menor oportunidade de exposição à infecções microbianas pode favorecer o aparecimento de asma. Em conformação com esta hipótese, a diminuição das doenças infecciosas orais na metade de século passado coincidiu com o aumento na prevalência de asma e outras doenças alérgicas. Esta observação levou alguns pesquisadores a especular que a exposição às bactérias orais, incluindo patógenos associados a doenças periodontais, como gengivite e periodontite, podem desempenhar um papel protetor no desenvolvimento de asma e alergia (ARBES et al., 2006; CARD et al., 2010). 
Assim, uma relação inversa ao que é encontrada nas infecções respiratórias tem sido observada quando analisado a presença de alergias respiratórias e asmas em pacientes portadores de patógenos periodontais específicos (FRIEDRICH et al., 2006). A sensibilização imunológica destes pacientes a estes organismos pode gerar efeitos positivos no controle de algumas enfermidades respiratórias como a asma, devido à diminuição da hiper-resposta imunoinflamatória induzida por alérgenos (CARD et al., 2010). Elevadas concentrações de anticorpos IgG no soro vistos em pacientes portadores de infecções por Porphyromonas gingivalis foram associadas a baixa prevalência de asma e rinite alérgica, enquanto que altas concentrações de anticorpo IgG para Aggregatibacter actinomycetemcomitans associou-se à respiração asmática (ARBES et al., 2006).

Entretanto, o conhecimento atual sobre a resposta imune às bactérias orais e patogênese imunológica das doenças periodontais vem sugerindo mecanismos biologicamente plausíveis pelos quais patógenos orais podem influenciar o risco de doença alérgica. A gengivite, comumente encontrada em crianças asmáticas, pode ser explicada pelas alterações na resposta imune e pela desidratação da mucosa alveolar devido à presença freqüente de respiração bucal. Em pacientes asmáticos, autores referenciam o aumento da concentração de IgE no tecido gengival, o que pode contribuir para o estabelecimento da destruição periodontal. Esta mesma concentração aumentada pode ser vista em estados de inflamação gengival mais grave em pacientes não asmáticos mesmo que os mecanismos de defesa imunológicos não se mostrem alterados (THOMAS et al., 2010). Assim, as reações de hipersensibilidade da asma poderão estar relacionadas com o processo de desenvolvimento da doença periodontal, onde os níveis de IgE encontram-se elevados no tecido gengival de um portador de periodontite. Interações entre bactérias e fatores imunológicos constituem-se então a principal causa de destruição periodontal (COSTA; XAVIER, 2008; SHASHIKIRAN; REDDY; RAJU, 2007).

Em um estudo que avaliou 80 pacientes portadores de asma brônquica demonstrou que, em comparação com grupo controle, onde aspectos relacionados à idade, sexo e nível socioeconômico foram pareados, os resultados mostraram um aumento significativo nos parâmetros clínicos de inflamação gengival entre os asmáticos comparado aos indivíduos saudáveis (MEHTA et al., 2009).

\section{Medicações para tratamento da asma e alterações no ambiente bucal}

As medicações mais comumente utilizadas por pacientes com asma incluem: ante-histamínicos, betaantagonistas e esteróides, de uso sistêmico ou inalados, prescritos de acordo à severidade da asma e presença ou não de co-morbidades. Estas medicações podem provocar xerostomia e diminuição do $\mathrm{pH}$ salivar (THOMAS et al., 2010). Em um estudo que comparou 65 crianças e adolescentes asmáticos a outros 65 não asmáticos mostrou que em pacientes com asma grave o risco de diminuição do fluxo salivar é 17 vezes maior quando comparado ao grupo sem doença. Entretanto, entre os indivíduos portadores de asma moderada e os não doentes, não foram observadas diferenças significantes (PAGANINI et al., 2011). A saliva exerce um importante papel de proteção imunológica contra patógenos bucais, bem como atua mecânica e quimicamente no controle da formação do biofilme, além de contribuírem para a manutenção fisiológica dos tecidos bucais (ERSIN et al., 2006; FRIEDRICH et al., 2006).

Considerados os medicamentos de escolha no tratamento da asma grave, os corticosteróides, tanto de uso oral como os inalados, atuam diminuindo a resposta inflamatória e deprimindo o sistema imunológico, através da liberação de ácido araquidônico, inibição da produção das citocinas próinflamatórias, diminuição da migração de neutrófilos para os locais de inflamação, redução da função das células $\mathrm{T}$ e diminuição do número de eosinófilos (SANTOS et al., 2007; SHASHIKIRAN; REDDY; RAJU, 2007). A absorção sistêmica pode ocorrer diretamente pela superfície pulmonar ou por deglutição da droga. Imediatamente após a inalação aproximadamente $10 \%$ a $20 \%$ da dose nominal liberada é depositada nos pulmões, enquanto a maior parte impacta-se na orofaringe e é deglutida. Seguindo-se a absorção a partir do trato gastrintestinal, a droga passa pelo fígado antes de entrar na circulação sistêmica (ALLEN et al., 2003). Alguns corticosteróides inalados, particularmente budesonida e fluticasona são metabolizados (89\% e $99 \%$ respectivamente) durante sua primeira passagem pelo fígado. Portanto, após a absorção oral, entram na circulação sistêmica como metabólitos inativos. A maioria das drogas, entretanto, não são eficientemente inativadas durante o primeiro passo do metabolismo e entram na circulação sistêmica sem modificações, resultando em efeitos colaterais extrapulmonares (D'OTTAVIANO et al., 2006).

Evidencias sugerem que os corticosteróides absorvidos pela circulação sistêmica podem provocar diminuição na densidade mineral óssea através de vários mecanismos (THOMAS et al., 2010). Eles determinam a redução da absorção intestinal de cálcio (um efeito antivitamina D) e consequente calciúria, que se seguem por aumento compensatório da produção do paratormônio (PTH) que vai determinar a mobilização do cálcio constituinte da massa óssea para a circulação. Além disso, na matriz óssea, o corticosteróide tem efeito supressor direto sobre o osteoblasto, o que reduz a formação óssea (MARTINATI et al., 1996; TOOGOOD et al., 1995). A exposição aos cortecosteróides altera o 
frágil equilíbrio entre a atividade dos osteoblastos e osteoclastos no metabolismo ósseo, ocasionando um maior estímulo dos osteoclastos na reabsorção óssea mediada pelos osteoblastos e redução na formação óssea, o que resulta em aumento da reabsorção óssea global. Especificamente, os dois principais efeitos dos corticosteróides no metabolismo ósseo são: (1) indução da apoptose de osteoblastos e osteócitos, e consequente diminuição da formação óssea, e (2) prolongamento da vida útil dos osteoclastos, o que leva a uma maior reabsorção óssea (MITRA, 2011).

O uso regular de doses convencionais de corticosteróides inalados por pacientes com asma pode suprimir a função adrenal e diminuir a densidade mineral óssea. A perda óssea sistêmica causada por esta droga, especialmente em usuários de altas doses por longos períodos de tempo, pode exercer um importante papel no desenvolvimento e progressão da doença periodontal (ERSIN et al., 2006; KELYY et al., 2008). Em um estudo com trinta e quatro crianças de 4 a 14 anos com asma persistente moderada ou grave, tratados com corticosteróides inalados há pelo menos um ano foram avaliados quanto à velocidade de crescimento, metabolismo ósseo e densitometria mineral óssea. Desta amostra, a alteração na densidade mineral óssea foi documentada em $32,3 \%$ dos pacientes e esteve associada à redução da velocidade de crescimento principalmente entre os indivíduos com formas mais graves da doença, mas sem relação com tempo de tratamento e dose cumulativa do corticosteróide recebido (D'OTTAVIANO et al., 2006).

Outras evidências sugerem que a perda de dentes em pacientes asmáticos que fazem uso de terapia com corticosteróides inalados pode estar relacionada à diminuição da densidade óssea, especialmente na mandíbula. Esses autores sugerem que, pacientes que fazem uso desta medicação por longos períodos de tempo façam avaliações periódicas da densidade mineral óssea mandibular, principalmente os que forem alvos de fatores de risco relacionados à osteoporose (HAN et al., 2009).

\section{COMENTÁRIOS FINAIS}

Apesar do crescente interesse da comunidade científica em relacionar as condições periodontais ao desenvolvimento de doenças sistêmicas, um dos principais entraves no que se refere ao estabelecimento de uma relação causal reside na falta de critérios de diagnóstico para doença periodontal. Em relação às condições de saúde bucal, não existe consenso entre os autores sobre a escolha de um melhor índice de avaliação e diagnóstico da periodontite, surgindo, desta forma, dificuldades na comparação dos resultados obtidos nos diversos trabalhos publicados.

Em relação às doenças pulmonares, a infecção periodontal parece agir de forma distinta quando são comparadas as doenças de caráter infeccioso, como as pneumonias, e as doenças respiratórias alérgicas e asma. Estudos com doenças respiratórias infecciosas parecem demonstrar uma forte associação entre a quantidade de placa bacteriana presente na cavidade bucal e sua associação com o desenvolvimento de pneumonias. Pacientes com pobre higiene oral podem abrigar em sua cavidade bucal os possíveis precursores de infecções pulmonares, que por aspiração, podem ser levados para o interior do trato respiratório. A depender do grau de defesa imunológica apresentado por esses indivíduos, o simples contato desses microrganismos com o epitélio respiratório, podem ser suficiente para o desenvolvimento de uma infecção pulmonar.

Entretanto, por ser a asma uma doença inflamatória, pacientes asmáticos possuem uma alteração nas capacidades de defesa do organismo, o que facilitaria o desenvolvimento de infecções, entre elas a doença periodontal. Uma vez que, a infecção periodontal é capaz de induzir o sistema imunológico do indivíduo a produzir citocinas importantes na degradação, não somente de bactérias patogênicas, como também do tecido do hospedeiro, e, associado ao fato dessas citocinas não agirem localmente, mas terem influência sistêmica faz-se necessário, portanto, pensar em mecanismos de avaliação do status de condição bucal de pacientes com asma. Nestes pacientes, a presença de patógenos periodontais pode estimular a produção de anticorpos específicos que poderiam alterar a hiper-resposta imunoinflamatória.

Outro fator importante a ser considerado diz respeito ao uso de corticosteróides por longos períodos de tempo para tratamento da asma. Estudos vêm demonstrando a capacidade dos corticosteróides de reduzir a defesa imunológica do indivíduo, bem como, exercer influencia no metabolismo ósseo, levando a sua menor mineralização. A redução do fluxo salivar apresentado por usuários desse tipo de medicação pode estar fortemente associada ao desenvolvimento da periodontite. Ora, se em indivíduos asmáticos, o desenvolvimento da doença periodontal pode ser mais expressivo quando comparado aos indivíduos saudáveis, a presença de um maior número de microrganismos orais pode contribuir para um pior controle da doença, bem como, favorecer infecções respiratórias. Ao mesmo tempo, a diminuição da qualidade óssea alveolar pode ampliar as chances de surgimento de lesões periodontais.

\section{CONCLUSÕES}

Todos os estudos aqui apresentados ratificam a necessidade de uma melhor investigação a respeito da interferência da atuação dos patógenos periodontais no sistema imune de pacientes com asma. Ao mesmo tempo, novos estudos são necessários para esclarecer de que forma o tratamento da asma pode influenciar no aparecimento ou agravamento da doença periodontal. 
Considerando-se que os dados apresentados nas pesquisas consultadas ainda não são concludentes, será de suma importância realização de novos trabalhos investigativos de caráter epidemiológico, por meio de pesquisas bem controladas.

\section{REFERÊNCIAS}

1. ALLEN, D. B. et al. Inhaled Corticosteroids - Past Lessons and Future. J. Allergy Clin. Immunol., St. Louis, v. 112, n. 3, p. S1S40, Sept. 2003.

2. ARBES, S. J. Jr. et al. Oral pathogens and allergic disease: results from the Third National Health and Nutrition Examination Survey. J. Allergy Clin. Immunol., St. Louis, v. 118, n. 5 , p. $1169-1175$, Nov. 2006

3. ARMITAGE, C. G. Development of a classification system for Periodontal Disease and conditions. Ann. Periodontol., Chicago, v. 4, n. 1, p. 1-6, Dec. 1999.

4. AZARPAZHOOH, A.; LEAKE, J. L. Systematic Review of the association between respiratory diseases and oral health. J. Periodontol., Indianapolis, v. 77, n. 9, p.1465-1482, Sep. 2006.

5. BÁGYI, K. et al. Role of pathogenic oral flora in postoperative pneumonia following brain surgery. BMC Infect. Dis., London, v. 9, p.104, Jun. 2009.

6. CARD, J. W. et al. Modulation of allergic airway inflammation by the oral pathogen Porphyromonas Gingivalis. Infect. Immun., Washington DC, v. 78, n. 6, p. 2488-2496, Jun. 2010.

7. COSTA, A. L.; XAVIER, T. Implicações da asma na saúde oral infantil. Acta Pediatr. Port., Lisboa, v. 39, n. 6, p. 260-265, nov./ dez. 2008.

8. D'OTTAVIANO, F. L. et al. Tratamento prolongado com corticosteróide inalado e o metabolismo ósseo em crianças com asma persistente. R. Bras. Alerg. Imunopatol., São Paulo, v. 29, n. 6, p. 263-267, nov./dez. 2006.

9. ERSIN, N. K. et al. Oral and dental manifestations of young asthmatics related to medication, severity and duration of condition. Pediatr. Int., Tokyo, v. 48, n. 6, p. 549-554, Dec. 2006

10. FRIEDRICH, N. et al. Inverse association between periodontitis and respiratory allergies. Clin. Exp. Allergy, London, v. 36, n. 4, p. 495-502, Apr. 2006.

11. GEMMELL, E.; CARTER, C. L.; SEYMOUR, G. J. Mast cells in human periodontal disease. J. Dent. Res., Washington, v. 83, n. 5, p. 384-387, May 2004.

12. GOMES-FILHO, I. S.; PASSOS, J. S.; CRUZ, S. S. Respiratory disease and the role of oral bacteria. J. Oral. Microbiol. Jarfalla, v. 2, p. 5811, Dec. 2010.

13. HAN, E. R. et al. Inhaled corticosteroid-related tooth problems in asthmatics. J. Asthma, New York, v. 46, n. 2, p. 160 164, Mar. 2009.

14. HUMBERT, M. et al. The immunopathology of extrinsic (atopic) and intrinsic (nonatopic) asthma: more similarities than differences. Immunol. Today, Cambridge, v. 20, n. 11, p. 528-533, Nov. 1999.

15. ISAAC. Steering Committee. Worldwide variations in the prevalence of asthma symptoms: the International Study of Asthma and Allergies in Childhood (ISAAC). Eur. Respir. J., Copenhagen, v.12, n. 2, p.315-335, Aug. 1998.

16. KELLY, H. W. et al. Effect of long-term corticosteroid use on bone mineral density in children: a prospective longitudinal assessment in the childhood asthma management program
(CAMP) study. Pediatric., Evanston, v. 122, n. 1, p. e53-e61, Jul. 2008.

17. MARTINATI, L. C. et al. Effect on cortical and trabecular bone mass of different anti-inflammatory treatment in preadolescents children with chronic asthma. Am. J. Respir. Crit. Care Med., New York, v. 153, n. 1, p. 232-236, Jan. 1996.

18. MATOS, A. P. S.; MACHADO, A. C. C. Influência das variáveis biopsicossociais na qualidade de vida em asmáticos. Psicol. Teor. Pesqui., Brasília, v. 23, n. 2, p. 139-148, abr./jun. 2007.

19. MEHTA, A. et al. Is bronchial asthma a risk factor for gingival diseases? A control study. N.Y. State Dent. J., New York, v. 75, n. 1, p. 44-46, Jan. 2009.

20. MITRA, R. Adverse effects of corticosteroids on bone metabolism: a review. PM R, Baltimore, v. 3, n. 5, p. 466-471, May 2011.

21. MORJARIA, J. B.; POLOSA, R. Recommendation for optimal management of severe refractory asthma. J. Asthma Allergy, Auckland, v. 3, p. 43-56, Jul. 2010.

22. PACE, C. C.; McCULLOUGH, G. H. The association between oral microorganisms and aspiration pneumonia in the institutionalized elderly: review and recommendation. Dysphagia, New York, v. 25, n. 4, p. 307-322, Dec. 2010.

23. PAGANINI, M. et al. Dental caries status and salivary properties of asthmatic children and adolescents. Int. J. Paediatr. Dent., Oxford, v. 21, n. 3, p. 185-191, May 2011.

24. PIZZO, G. et al. Dentistry and internal medicine: from the local infection theory to the periodontal medicine concept. Eur. J. Intern. Med., Amsterdam, v. 21, n. 6, p. 496-502, Dec. 2010.

25. SANTOS, N. C. N. et al. Efeitos relacionados ao uso do esteróide inalado na saúde periodontal que o médico precisa conhecer. R. Bras. Alerg. Imunopatol., São Paulo, v. 30, n. 6, p. 220-226, nov./dez. 2007.

26. SCANNAPIECO, F. A.; DASANAYAKE, A. P.; CHHUN, N. Does periodontal therapy reduce the risk for systemic diseases? Dent. Clin. North Am., Philadelphia, v. 54, n. 1, p. 163-181, Jan. 2010.

27. SCANNAPIECO, F. A. Role of oral bacteria in respiratory infection. J. Periodontol., Indianapolis, v. 70, n. 7, p. 793-802, Jul. 1999.

28. SHASHIKIRAN, N. D.; REDDY, V. V.; RAJU, P. K. Effect of antiasthmatic medication on dental disease: dental caries and periodontal disease. J. Indian Soc. Pedod. Prev. Dent., Chandigarh, v. 25, n. 2, p. 65-68, Apr./Jun. 2007.

29. SIGUSCH, B. et al. Early-onset and adult periodontitis associated with abnormal cytokine production by activated $\mathrm{T}$ Iymphocytes. J. Periodontol., Indianapolis, v. 69, n. 10, p. 10981104, Oct. 1998.

30. SOCRANSKY, S. S.; HAFFAJEE, A. D. The bacterial etiology of destructive periodontal disease: current concepts. J. Periodontol., Indianapolis, v. 63, n. 4, p. 322-331, Apr. 1992.

31. THOMAS, M. S. et al. Asthma and oral health: a review. Aust. Dent. J., St Leonards, v. 55, n. 2, p. 128-133, Jun. 2010.

32. TOOGOOD, J. H. et al. Bone mineral density and the risk of facture in patients receiving long-term inhaled steroid therapy for asthma. J. Allergy Clin. Immunol., St. Louis, v. 96, n. 2, p. 157-166, Aug. 1995

33. VAN DYKE, T. E. Cellular and molecular susceptibility determinants for periodontitis. Periodontol. 2000, Copenhagen, v. 45, n. 1, p. 10-13, Oct. 2007. 
34. ZANATTA, F. B. et al. A participação das Células T Helper na Periodontite Crônica. R. Periodontia, Rio de Janeiro, v. 19, n. 2, p. 7-13, jun. 2009. 